\title{
LA SÉPARATION BRUTALE : LE SUICIDE ET L'ÉCLATEMENT FAMILIAL DANS QUELQUES ROMANS QUÉBÉCOIS CONTEMPORAINS
}

Molleen Shilliday, Université de Colombie-Britannique

Selon Emile Durkheim, les rapports entre l'individu qui se tue et «la société religieuse, domestique ou politique » dont il fait partie révèlent les causes du suicide. ${ }^{1}$ En effet, les cinq romans à l'étude, L'Ingratitude de Ying Chen, ${ }^{2}$ Unless de Hélène Monette, ${ }^{3}$ L'Île de la Merci d'Élise Turcotte, ${ }^{4}$ Une folie sans lendemain de Nicole Houde $^{5}$ et Le Fou de Bosch de Sergio Kokis, ${ }^{6}$ examinent tous le rôle de la société domestique, c'est-à-dire de la famille, dans la vie du personnage qui se suicide. Ils explorent l'idée que I'hérédité, l'enfance et la mémoire fondent la base de l'histoire personnelle et soulignent que ces éléments sont inextricablement liés à la conception de la cellule familiale (ou à l'absence de celle-ci dans le cas de l'orphelin). L'histoire personnelle forme l'identité du personnage qui se suicide et influence ses décisions. D'ailleurs, lorsque nous considérons le niveau d'interdépendance psychologique entre le personnage et sa famille ainsi que les émotions fondamentales (I'amour et la haine) sur lesquelles cette interdépendance est fondée, les relations familiales s'avèrent d'une importance singulière.

La question de I'hérédité est explorée le plus explicitement dans Unless. Après le suicide de leur frère par coup de carabine, les sœurs de Chut, Unless, Milou et Red, se remémorent leurs souvenirs d'enfance et interrogent leur histoire familiale pour, enfin, trouver la source de leur souffrance et de leur désarroi. C'est à travers cette enquête qu'elles découvrent qu'elles peuvent en condamner leur mère disparue. En effet, le suicide de Chut soulève le débat, à jamais inexhaustible, de l'inné et de l'acquis. D'après Baechler, tandis qu'il est possible qu'un goût pour le suicide soit transmis héréditairement, il est probable qu'il constitue plutôt un réflexe conditionné, une technique que l'enfant apprend de son parent pour éviter l'angoisse de la vie. ${ }^{7}$ Par contre, il est tenu pour acquis que certaines maladies d'âme et de corps et, parfois, la disposition de l'individu (par exemple, une tendance à la mélancolie) proviennent des ascendants. Il semble clair que Chut ait hérité de la folie de sa mère. Pourtant, pouvons-nous mettre en cause une mère folle et absente pour le suicide de son fils ? Les sœurs s'acharnent à trouver un lien entre la folie de la mère, son départ qui s'en suit, et le suicide du fils fou. L'existence d'un rapport entre ces deux événements devient irréfutable lorsque nous analysons la réaction d'Unless au suicide :

Il voulait me tuer mais il a essayé la carabine avant. Quand? Tu n'as rien à voir là-dedans! Maman? Non. Pas de plumes. Que du sang. Non. Pas de mère. [...]Faites signer quelqu'un pour les électrochocs. Elle aurait pas dû avoir des enfants (U 56). ${ }^{8}$

Pour Unless, le suicide de son frère évoque inévitablement les souvenirs traumatisants de la folie et de la disparition de la mère. La description de cette scène est marquée par une rupture temporelle entre ces deux moments de perte familiale; le lecteur n'a aucun point de repère pour les différencier l'un de l'autre. D'ailleurs, notons que le style fragmentaire prend ici son essor et, donc, mime le désarroi que ces deux événements tragiques 
ont provoqué. L'histoire de la mère et celle du fils s'interpénètrent et révèlent la force complexe qui les relie. Selon Oore, puisque Chut se suicide au sous-sol, le même endroit d'où sa mère est partie, les deux moments deviennent «irréversiblement liés ». Elle note que l'endroit du suicide rappelle «les lieux sombres, profonds, enfoncés [qui] sont des archétypes de la matrice maternelle ». ${ }^{9}$ Bien que I'omniprésence de la figure matriarcale soit manifeste depuis le début du roman, après la mort de Chut, le va-et-vient entre le passé et le présent s'amplifie mettant l'accent sur le fait que les autres enfants d'Adélaïde seront également incapables d'échapper à son influence. Dorénavant, la question perpétuelle devient: Qui sera la prochaine à être atteinte de folie, à succomber à la tentation de renoncer à la vie ?

Tout comme dans Unless, la figure matriarcale représente un point de départ pour l'exploration de I’histoire familiale dans Une folie sans lendemain. Le récit se déroule dans deux espaces-temps. Dans le présent, Céline, la narratrice, est informée par son médecin qu'elle est atteinte d'un «cancer foudroyant » (FSL 16). Face à sa propre mort, Céline rédige un mémoire consacré au souvenir de son enfance qui l'a toujours hanté : le jour où elle a découvert sa mère pendue dans le hangar. C'est à travers la remémoration de cette époque de sa vie qu'elle prend conscience de l'identité véritable de sa mère : elle était sensible à la souffrance des autres, spirituellement liée à la terre et complètement libre. Un texte remarquablement dur et triste, ce roman est sous-tendu par une pulsion libératrice. Edmée, se suicide pour tuer la folie en elle, son histoire nous permet de constater ce que le suicide d'une mère peut apprendre à ses descendants.

Je sentais s'imprimer dans mon cou le chanvre tressé, la corde se resserrait, une image m'avalait et coupait ma respiration. À quatorze ans, j'avais découvert ma mère pendue dans le hangar, derrière la maison. Puis, toujours, ma mère se dressait devant moi, le visage violacé, le cou rompu, les jambes ballantes. (FSL 13)

Ce passage, qui se trouve au début du roman, annonce l'importance du thème de la non-séparation corporelle de la mère et de la fille. Elle revit ce moment tragique comme s'il s'agissait de son propre suicide. Il est manifeste que ce souvenir la hante et influence directement la construction de sa propre identité. Tout comme les sœurs dans Unless qui sont 'ensorcelées ${ }^{10}$ par la mémoire de leur mère, Céline est prisonnière de ses souvenirs d'enfance, de la folie de sa mère et de son suicide. Dans un roman, comme dans l'autre, les filles explorent leurs souvenirs d'enfance pour enfin cerner chacune son identité. Le sentiment d'enfermement qu'éprouve Céline coïncide avec le concept du corps de la mère qui est partagé par la fille. Au cours du roman, il devient clair que Céline a, d'une certaine manière, hérité de l'altérité de sa mère ; les autres membres de la communauté la considèrent comme étant une extension de sa mère, une « autre » qui apportera de la mauvaise fortune. En racontant I'histoire de sa mère, Céline se libère de la hantise de la «mère pendue » et opère une sorte de rehaussement de la mère de son statut inférieur $\mathrm{d}^{\prime} \ll$ autre .

La prosopopée L'Ingratitude a également pour thème central les répercussions d'une relation trop intense entre mère et fille. Nous sommes bouleversés par le style du texte : c'est par le regard d'une morte, Yan-Zi, que 
nous voyons le monde, alors que celle-ci flotte au-dessus de ses pairs dans un espace entre-deux. Lorsqu'elle nous raconte son cheminement vers la mort, elle remet en cause les attentes familiales et les contraintes sociétales. Elle assume son ingratitude et affirme son droit à l'autonomie. Selon Yan-Zi, son suicide constitue une vengeance contre sa mère ainsi qu'un rejet du contrôle qu'exerce la société chinoise sur elle en tant que jeune femme nonmariée. Le père est caractérisé comme étant faible d'esprit et indifférent à sa famille, la mère, donc, prend les rôles de matriarche et de patriarche. La mère déteste sa fille parce qu'elle la voit comme étant différente d'elle-même ; d'autre part, la fille hait sa mère car celle-ci refuse de lui accorder le droit à sa propre identité. Par conséquent, leur relation se nourrit de cette haine mutuelle qui se manifeste dans leur conduite et à travers leurs paroles : la mère menace de «battre [sa fille] à mort » (I 84); la fille imite la violence de la mère en déclarant qu'elle est « prête à [s]'empoisonner ou à étrangler cette femme » (I 84). La mère menace de se suicider (I 91) ; la fille planifie son propre suicide. En effet, leur violence se fait écho, soulignant à la fois leur interdépendance et la nécessité de leur séparation. Le seul moyen pour Yan-Zi d'accomplir cette séparation, de devenir autonome, est à travers la violence : «Nous avions besoin d'une séparation brutale, d'un déracinement féroce pour sortir de la torpeur et nous redécouvrir, sinon pour nous abandonner définitivement » (I 12). Il est intéressant de noter que la «séparation brutale» dans ce passage évoque la violence de l'accouchement. En effet, les thèmes d'accouchement, d'accouchement d'un mort-né ou d'avortement ${ }^{11}$, voire de maternité violente, reviennent comme un leitmotiv dans le roman et mettent l'accent sur l'idée que l'identité de la fille est inséparablement liée à sa conception dans le ventre de sa mère. «Si j'avais pu choisir, j'aurais préféré mourir dans la chaleur discrète du corps maternel » (I 23). Alors que, pour la fille, le corps de la mère représente la mort, pour la mère, le corps de la fille signifie sa renaissance : « J'étais chargée de porter en moi l'esprit de maman dont le corps pourrirait tôt ou tard. » (I 96-97). Au lieu de tenter de connaître sa fille, la mère semble croire qu'il est plus simple de la voir comme la « reproduction » (I 97) d'elle-même. En se suicidant, Yan-Zi essaie de se libérer de cette notion de reproduction : «Il fallait tuer sa fille. Il n'y avait pas d'autres moyens de la rendre plus sage. Je ne pouvais pas être moi autrement » (I 97). La fille se tue afin de se séparer définitivement de sa mère, pour affirmer son moi.

Dans L'T̂le de la Merci, Élise Turcotte dresse le portrait de la famille nucléaire ; Hélène, Lisa, Samuel et leurs parents, Viviane et Robert, occupent une maison blanche de la banlieue. Pourtant, au fur et à mesure que I’histoire progresse, la narratrice, Hélène, dévoile les fausses apparences et révèle une vie de carence parentale et de souffrance dissimulée. C'est une existence immobile et silencieuse : la nécessité d'un incident navrant pour qu'ils reviennent à la vie semble manifeste. Puisque Hélène est obsédée par la violence sociétale et réagit contre les déceptions qu'elle subit de la part de ses parents par un comportement insoumis et violent, le lecteur croit que ce sera elle qui sera l'instigatrice d'un changement violent et définitif. Cependant, c'est Lisa, la douce rêveuse, qui ose commettre l'injure finale aux parents lorsqu'elle se suicide. Le premier chapitre intitulé «La Prison » s'ouvre sur la description de la maison familiale. Il est clair que l'écrivaine crée un lien métaphorique entre ces deux 
endroits. À la première page, nous rencontrons Hélène, seule dans sa chambre «nette et lisse » qu'elle compare à son corps : «L'intérieur de son corps doit être ainsi : une chambre carrée contenant des formes géométriques invariables. Un lit, une commode, une bibliothèque. Pas de saleté. Rien de criant» (IM 13). Cette image évoque une cellule de prison et implique que l'emprisonnement physique qu'elle se croit en train de vivre affecte son état psychologique. Sa chambre est au deuxième étage et telle une prisonnière, elle regarde par la fenêtre et rêve d'une vie fictive :

Une jeune fille pourrait s'arrêter devant la maison et crier: «Es-tu prête ? ; Hélène pourrait alors dévaler l'escalier, sortir, et enfourcher sa bicyclette. Mais ce n'est pas ça. Rien n'est vraiment joyeux, et rien n'est encore mort. Tout est seulement un peu parti, disparu, délavé. (IM 13-14)

L'écrivaine crée un espace romanesque isolé et statique. D'un côté et de l'autre la maison est séparée du monde extérieur par des obstacles tels que la rivière, l'île et la voie ferrée soulignant le motif d'enfermement. D'ailleurs, nous apprenons la proximité de « la prison Bordeaux» ce qui cimente le rapport métaphorique entre la maison et la prison. Dans ce roman, la relation entre les parents et les enfants est dépourvue de l'amour dont les enfants ont besoin pour se sentir intégrés dans le contexte familial. L'esprit qui règne est celui de « chacun pour soi » ; cette séparation « des êtres » constitue, aux yeux des enfants, un rejet impardonnable. Alors que ce rejet provoque des crises de colère et de violence chez Hélène et chez Samuel, Lisa se recroqueville, gardant sa souffrance pour ellemême. L'attention parentale insuffisante ainsi que l'agression qui découle de la détérioration progressive du mariage de Viviane et de Robert pèsent lourdement sur Lisa. Par ailleurs, le dysfonctionnement familial mène les membres de la famille à ignorer les signes précurseurs du suicide. Au lieu d'extérioriser la frustration qu'elle ressent, comme le fait sa sœur, Lisa I'intériorise jusqu'au jour où elle implose en se tuant au grenier. En effet, la mère rénove le grenier pour s'y créer un lieu de refuge ; elle interdit aux enfants d'y mettre les pieds. Sur le plan symbolique, le grenier s'édifie comme une manifestation visible de la distance que la mère met entre elle et ses enfants. Tout comme pour Chut dans Unless, le choix du lieu du suicide rend la mère coupable explicitement.

Atteint de folie et désireux de fuir l'anonymat de son lot d'orphelin, le personnage principal du Fou de Bosch de Sergio Kokis, Steiner, s'imagine comme étant la figure centrale des tableaux de Jérôme Bosch : le Christ. Nous le suivons, lorsqu'il parcourt I'Europe cherchant les traces d'un père mythique et se délectant des tableaux de son maître. Sa folie finit par l'envahir complètement et il se noie en chantant le «mantra » (FB 223) qui l'a toujours bercé dans ses moments de dépression extrême: « My daddy lies over the ocean, My daddy lies over the sea...Bring back, Bring back, Bring back my daddy to me... » (FB 223). ${ }^{12}$ Il est clair que l'errance et le vagabondage de Steiner sont provoqués par l'absence d'une identité définie et le rêve d'une autre existence toujours hors de portée, c'est-à-dire, celle de la vie familiale. Le mystère autour de son histoire personnelle hante Steiner et influence ses croyances fondamentales ainsi que ses actions les plus significatives. Il bute toujours contre l'idée que parce qu'il a été abandonné, il est inférieur. Selon lui, les autres « ri[ent] de sa bâtardise » (FB 106). Puisqu'il ramène toujours le passé au présent, les déceptions qu'il a vécues pendant son enfance finissent par le définir : il 
est orphelin abandonné et dépourvu de l'identité concrète qui provient d'une histoire familiale. Il est nécessaire de commenter ici la signification symbolique de sa vision de lui-même comme étant le Christ de Bosch. Le Christ est le Messie, le fils de Dieu. Or, le Christ est l'inverse de l'orphelin. Il représente ce que Steiner a toujours convoité : être le sujet de la vénération du père et d'autrui. Il est clair que Steiner est déboussolé par l'absence du père. Il n'a pas de critères sur lesquels il peut baser son identité. Bachler stipule la personnalité de l'orphelin « est mal rassemblée, sans ossature ni ligne de force ${ }^{13}$ En effet, Steiner invente une histoire familiale afin de compenser son identité déficiente. Il se dit que son père était marin et que sa mère était une prostituée. Il se sent toutefois au bord d'un gouffre, «avalé » par ses déceptions d'enfance. Ainsi, veut-il punir métaphoriquement ses parents de l'avoir abandonné, en les imaginant souffrir dans les tableaux de Bosch qui dépeignent toujours la souffrance humaine et la condamnation des pécheurs.

Voilà : naufragé et avalé par un monstre des abîmes. Et puis, droit en enfer sans autres précisions, comme il a vécu. [...] Avalé par un poisson, ce n'est que justice. Est-ce que moi, je n'ai pas été avalé par l'orphelinat? Alors, crève! (FB 85). ${ }^{14}$

Il est intéressant de noter que cette digression dans l'imaginaire s'enracine dans l'inconscient du fou; avec le temps, elle se transforme en vérité pour Steiner. En effet, à la fin du roman, il se noie pour sauver son père de ce poisson fictif qu'il a lui-même inventé. La perte d'un parent, par la mort ou par une séparation définitive, mène l'enfant à vouloir rejoindre celui qui est parti. Steiner peut, donc, rationaliser son désir de se tuer, puisqu'il va finalement rejoindre ce père mythique.

L'histoire personnelle ainsi que les relations interfamiliales s'avèrent des causes importantes du suicide dans le roman québécois contemporain. Alors que les personnages adultes sont hantés par le passé et leurs souvenirs d'enfance, les jeunes rêvent d'une meilleure situation familiale. Il est important de noter qu'alors que le récit du Fou de Bosch se termine au moment même du suicide, dans Unless, dans Une folie sans lendemain, dans L'Ingratitude et dans L'île de la Merci, après le suicide, l'éclatement familial se voit remplacé par le renouvellement et la reconstruction pour ceux et celles qui survivent au personnage qui se suicide. Le trajet narratif rétrospectif d'Une folie sans lendemain, qui nous permet d'examiner le rapport entre la mère et la fille une quarantaine d’années après le suicide, élabore la notion de la reconstruction plus amplement que les autres romans. Alors que le souvenir du suicide de sa mère a hanté Céline sa vie durant, lorsqu'elle fait face à sa propre mort, elle réalise que la remémoration de la pendaison de sa mère lui apporte la liberté. La reconstruction ne peut se faire donc sans la remémoration, il faut faire face à l'impasse, investir notre énergie à la fois dans le passé et dans le présent, pour pouvoir affirmer la vie même au cœur de l'éclatement.

${ }^{1}$ Durkheim, Le Suicide: étude de sociologie, 222.

${ }^{2}$ Ying Chen, L'Ingratitude, Montréal : Leméac, 1995. Toutes références à ce roman seront incorporées au texte à I'aide du sigle I suivi du numéro de la page. 
${ }^{3}$ Hélène Monette, Unless, Montréal : Boréal, 1995. Toutes références à ce roman seront incorporées au texte à I'aide du sigle $U$ suivi du numéro de la page.

${ }^{4}$ Élise Turcotte, L'île de la Merci, Ottawa : Leméac, 1997. Toutes références à ce roman seront incorporées au texte à l'aide du sigle IM suivi du numéro de la page.

${ }^{5}$ Nicole Houde, Une folie sans lendemain, Montréal : Pleine Lune, 2002. Toutes références à ce roman seront incorporées au texte à l'aide du sigle FSL suivi du numéro de la page.

${ }^{6}$ Sergio Kokis, Le Fou de Bosch, Montréal : XYZ, 2006. Toutes références à ce roman seront incorporées au texte à l'aide du sigle FB suivi du numéro de la page.

${ }^{7}$ Baechler, Les Suicides, 340.

${ }^{8}$ En italique dans le texte.

${ }^{9}$ Oore, « Etre ou ne pas être: le suicide dans L'Ingratitude de Ying Chen, dans Unless d'Hélène Monette et dans L'Île de la merci d'Élise Turcotte » dans Dalhousie French Studies, 48.

10 «Qui étais-tu, Adélaïde, pour être à ce point ensorcelante à présent que les temps révolus me reviennent? 》 (U 30).

11 « Si je t'avais connue avant ta naissance, me disait-elle, je me serais fait avorter! » (I 109)

12 En italique dans le texte.

${ }^{13}$ Baecher, 343.

${ }^{14}$ En italique dans le texte. 